\title{
A Novel Technique for Fabricating Metalized Objects with Difficult Geometries
}

\author{
Mitch Danger Powers \\ UW-Madison Observational Cosmology
}

\begin{abstract}
In the course of fabricating corrugated horn antennae a technique was developed to avoid certain geometric difficulties. Stereolithograpy, a form of 3D printing, had been employed to create a lightweight, cheap, plastic horn. However, it was found that it was difficult to plate metal into the corrugations of the plastic horn. To work around this, a technique was developed where in the corrugated horn would be produced by assembling a number of easy to plate, interlocking rings. The rings would be plated separately, assembled, and electroplated with an exoskeleton. The general technique could readily be applied to a large number of similarly difficult scenarios.
\end{abstract}

\section{Background}

The genesis of this project is in the QUBIC bolometric interferometer. In order to save weight and manufacturing costs, the profiled corrugated microwave horn antennae were made of plastic via a process known as Stereolithography (SL). They would then be electrolessly plated with a thin layer of nickel. Plating proved to be quite challenging, due to the aspect ratio and small scale of the corrugations. As the object of study would be the cosmic microwave background radiation, they were designed to receive radiation in the W-band $(75-110 \mathrm{GHz}, 3-4 \mathrm{~mm})$. This requires slots about a millimeter deep and half as wide to line the interior of the horn (Timbie, 2011). The nickel coat that was electrolessly deposited became "spotty" and of visibly low quality in the grooves. As SL had already been used to get around horn fabrication issues, it was looked to as being a potential solution to this problem.

Stereolithography. In a word, SL is amazing. It is a relatively young, and rapidly expanding area of 3D printing. It works fairly simply. A vat of plastic resin is selectively solidified, layer by layer, via a computer controlled laser. The precision available is stunning, a state of the art machine is capable of printing $16 \mu \mathrm{m}$ layers, accurate to about $.025 \mathrm{~mm}$ and the minimum feature size is down around $.25 \mathrm{~mm}$. This makes SL an excellent candidate for millimeter regime component fabrication. In addition to the already impressive abilities of SL, it is also of note that it is comparatively cheap (prices scale by volume, and not complexity; a 3" long horn costs about two or three hundred dollars), lightning fast (parts can ship the same day they're ordered), and produces lightweight products (densities vary, but tend to about $1.3 \mathrm{~g} / \mathrm{cm}^{3}$ and components can have their interiors "honey-combed" to further cut weight), and designs that would otherwise be nearly, or in some cases fully, impossible to build become trivial (Quickparts). Further, the SL process culminates with a somewhat surreal moment where the machine operator can reach into the vat of resin and pull out a fully formed widget. The only

The author would like to thank WSGC for material support, as well as Peter Timbie for his guidance and expertise along the way. 
drawback I've encountered with SL is that they are yet to invent a conductive resin, and therefore any components that would need to be conductive require electroless plating.

Plating Plastic. While modern plastics are engineered to have a wide array of properties, to my knowledge we are yet to invent a conductive plastic. Adding a thin layer of conductive metal fixes this problem but creates one of its own, getting the metal to adhere to the plastic. Electroless plating works by mechanically anchoring a seed layer to the substrate, and plating, often auto-catalytically, onto that. To make this possible, the plastic substrates require a series of acid based treatments to roughen the surface. This is

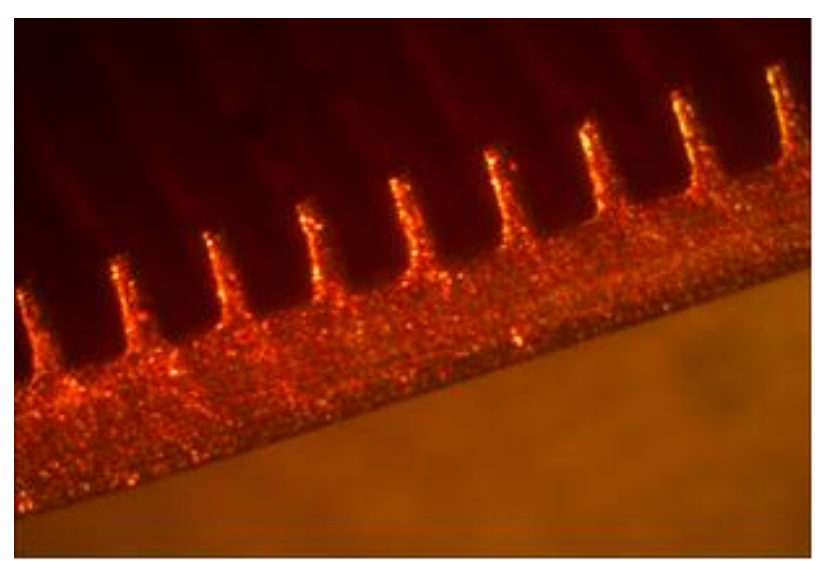

A section of electrolessly copper plated horn. This was produced prior to the switch to the Zhou electroless nickel process, which is considered to be an improvement. Timbie, 2011. followed by baths of stannous chloride and palladium chloride, which serve to "activate" the substrate and enable and catalyze the electroless deposition (Zhou, 2007). From there an off-theshelf electroless nickel plating solution provides a uniform, fine grained, conductive Nickel coating. This layer, which for the purposes of this project is made to be approximately $1 \mu \mathrm{m}$ thick, features a resistivity that tends to be roughly thrice that of bulk Nickel (thin film resistivity was measured to be approximately $4 \times 10^{-7} \Omega \mathrm{m}$ ). The nickel layer extends into holes and around corners exceptionally well, except for in cases of difficult geometries where the solutions have trouble reaching certain areas due to surface tensions, attack angles, and a host of other minor issues that become considerably more dominant in areas of difficult geometry.

\section{Design and Experimentation}

Rather than fight troubled areas, I have opted to remove them. The monolithic SL horn was deconstructed into a number of small interlocking rings, which, on their own, are remarkably easy to plate and are without difficult trenches.

a)

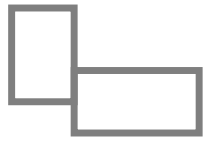

b)

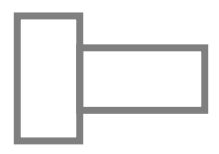

c)

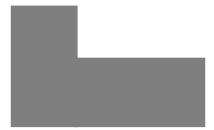

Ring Design. Several ring designs were considered, each with their own merits. The desired properties were that they be fairly simple, connect together with little or no seam, are stackable, and fit within SL capabilities. Shown at left are three of the considered ring cross sections. Ring A was ultimately chosen as it avoided any overly complex features, is readily stackable, and hides the ring-ring seam. Rings B and C are both very simple, but lack in stackability and feature prominent seams. More complicated designs involving multiple types of rings being stacked in order were scrapped because, while they hid all their seams and were extremely secure

Several prototype ring designs. See text. 
when stacked, they were overly complicated and tested some of the extremes of the SL machines.

Ring A is ideal for corrugated waveguides, where there is constant slot depth and width over a large stretch of waveguide. While the rings fit together nicely, and are easily adjusted to fit with the taper of a conical or even a profiled horn, incremental shifts in slot depth create an assembly issue. To avoid this, horn rings will be produced as an "exploded" horn, and attached to a SL rod that will keep them ordered until stacking (see cartoon at right). At which point they will be summarily broken off and stacked in their respective order.

Procedure. There are four distinct steps to the complete assembly process. The rings must be roughened, nickel plated, stacked, and then copper plated. For a detailed explanation of both the roughening and nickel plating process, the Zhou paper ought to be consulted, what is important though is the affect the roughening has on the later steps. The nickel coat creates a uniform enough layer that there

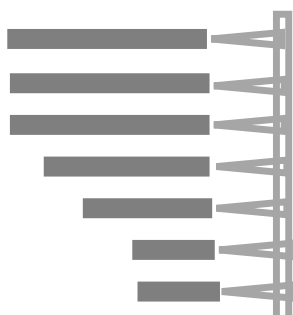

Horn rings will be kept ordered via a SL guide which will be removed after nickel plating. This is expected to reduce assembly time considerably. are no overt signs of deformity once the rings are plated. An overly rough ring would potentially create issues with ring to ring connections. A loose ring, or one with an acid puckered concave edge would introduce unwanted gaps into the stacked assembly, while a bloated, convex edge would damage the copper exoskeleton.

Stacking the nickel coated rings is precisely as simple as one would expect. So long as the rings are designed to mate with each other and nickel plated uniformly they connect together without issue. To ensure this, an entire assembly of rings ought be moved from bath to bath together. At times there is some resistance to stacking, especially if the rings are moist. To overcome this, rings should be left to dry in a clean, dry environment between processes. Further stacking issues can be overcome by applying a small amount of force along the z-axis of the horn. This can be delivered in any number of ways to fit the situation, however, I am quite fond of using a pen spring attached to a small plastic " $X$ " at each end of an assembly to hold it in place (if no such object is readily available, one might consider making a SL item to do the trick).

The copper exoskeleton binds the entire assembly is perhaps the most important step. It is put in place by a simple copper sulfate based electroplating system. For conductivity puposes, the exoskeleton would be required to several skin depths, $\delta_{\mathrm{s}}$, where $\delta_{s} \approx \sqrt{\frac{\rho}{\pi f \mu_{0} \mu_{r}}}, \rho=$ resistivity, and $\mu_{\mathrm{r}} \approx 1$, which works out to approximately $3 \mu \mathrm{m}$ in the microwave regime (Edwards, 1981). For structural purposes the exoskeleton would likely be several times thicker.

Due to unforeseen events, experiments on this stage of the process have been limited. However, it has been found that several items of nickel can be bound by a copper exoskeleton. Several nickel coated SL items, held lightly together - in this case by a piece of masking tape outside of 
the electroplating solution - and plated via a bath composed of copper sulfate and commercially available additives (Caswell Copper Electroplating Kit) with a 1 amp, 9 volt DC current forms an layer of copper that transcends substrate boundaries and forms an exoskeleton. It was observed that even as a thin layer, the copper made qualitative, obvious, improvements in the binding between nickel items. The copper layer held against the forces of gravity, but was inevitably torn asunder by a modest application of force.

Sadly, no quantitative data has been gathered on the properties of the exoskeleton. It has been shown to have obvious and beneficial affects even as a notably thin and low quality shell. Further experiments will create copper layers in excess of $10 \mu \mathrm{m}$ thick, perhaps thicker if mechanical issues demand it. This will serve as plenty thick for strong conductivity without interfering terribly with the overall dimensions of the final product.

\section{Applications}

Given even the modest performance of the copper plating, there is the possibility for a broad range of applications. In the specific case of a corrugated horn, not only can the entire bulk be assembled, but a traditionally manufactured portion of a horn can be fitted to an assembly of SL rings. Specifically, the throat section of the horn, which is perhaps the most crucial and most complicated section, can be electroformed as a solid, high quality piece, and an assembly can be mated with it by way of an overlapping exterior sheath, and the two can be electrobonded together. An existing fixture, perhaps a proprietary item that cannot readily be reproduced, can be fitted to a device with minimal effort. In more general situations, the technique can be used to produce a wide array of widget with specific thermal, electrical, or mechanical properties can be readily fabricated in house. This would be especially useful in a situation where new supplies are difficult to come by. A hypothetical mars colonist could be self-sufficient with little more than a SL machine, a small chemistry set, and a creative imagination.

\section{Future Work}

There are several stages of work that have been uncomfortably delayed to this point due to temporary circumstances. The copper coating requires several quantitative measurements to determine the resistivity of the coating, as well as measurements of the mechanical strength of the shell as affected by the copper thickness. Beyond that, a length of straight, corrugated waveguide will be constructed and tests of signal loss will be done in an anechoic chamber. Beyond that, it is hoped that the technique will be able to be applied to building a profiled corrugated horn antenna, with the tests that accompany that. Several gadgets may also be built in parallel to this work.

The time required to construct these devices is expected to be considerably short. As work has been delayed several times, design and theory have been developed considerably. Barring any further unexpected delays, it is expected that a waveguide will be constructed within a week of work resuming. 


\section{References}

Clarricoats, Peter John Bell., and A. David. Olver. Corrugated Horns for Microwave Antennas. London: P. Peregrinus, 1984. Print.

Edwards, T. C. Foundations for Microstrip Circuit Design. Chichester: Wiley, 1981. Print.

"Stereolithography (SLA) | Rapid Prototyping | Quickparts.com." Stereolithography (SLA) | Rapid Prototyping | Quickparts.com. N.p., n.d. Web. 9 Aug. 2012. <http://www.quickparts.com/LowVolumePrototypes/SLA.aspx>.

Zhou, Z., D. Li, J. Zeng, and Z Zhang. "Rapid Fabrication of Metal-coated Composite Stereolithography Parts." Proc. IMechE 221B: J. Eng. Manuf. 221.9 (2007): 1431-440. Print. 